\title{
Pandemia global, COVID-19 y podología
}

\author{
Global pandemic, COVID-19 and podiatry
}

En el momento de escribir esta carta editorial (23:00 horas; 6 de mayo de 2020) el número confirmado de infectados por COVID-19 a nivel mundial es de 3.732.046 y las muertes ocasionadas por la pandemia ascienden a un total de 261.517, siendo España uno de los países más afectados ${ }^{1}$. La declaración del estado de alarma en nuestro país conllevó como principal medida la imposición de una cuarentena nacional que entró en vigor a las 0:00 horas del domingo 15 de marzo como una de las medidas de emergencia para reducir el contagio. Como es evidente, nuestra profesión también se ha visto fuertemente afectada por toda esta situación.

A raíz de esto, hemos vivido un debate intenso en nuestra profesión sobre la necesidad de permanecer abiertos prestando asistencia podológica a los pacientes que lo necesitan por un lado, y el peligro que supondría no cerrar completamente nuestras clínicas durante el periodo de confinamiento por otro lado. Este debate ha estado alimentado principalmente en las redes sociales con opiniones diversas a favor de una u otra opción. El Consejo General publicó una nota de prensa, con fecha de 31 de marzo, en la que explicaba que los podólogos, como profesionales sanitarios, atenderían todas las urgencias podológicas en sus centros siempre y cuando se pudiera garantizar la seguridad de los pacientes y profesionales. Es un equilibrio complicado en el que se encuentra la necesidad de controlar la pandemia y de evitar visitas y desplazamientos innecesarios que ayuden a propagar la infección. Sin embargo, existe también la necesidad, como profesionales sanitarios, de asumir la responsabilidad de ser un servicio esencial para la sociedad y necesario incluso en esta situación. Personalmente, llevo año y medio trabajando como podólogo en la Unidad de Pie Diabético del Hospital de Cruces (Bilbao), que está adscrita al servicio de Cirugía Vascular y Angiología de dicho hospital, y puedo dar fe de la importancia y necesidad de prestar servicio podológico para este tipo de pacientes incluso en esta situación. Este tipo de pacientes, especialmente cuando se acompañan de complicaciones como enfermedad vascular periférica y/o neuropatía periférica, representan una población especialmente frágil con un riesgo muy elevado de pérdida de la extremidad, o incluso muerte, si no se lleva una monitorización cercana de sus pies por parte del podólogo 2,3. Los pacientes más complejos con grados más avanzados de isquemia, que presentan ulceraciones activas, o sin ellas pero sin posibilidades de recibir un cuidado rutinario periódico, también aumentan drásticamente el riesgo de infectarse, lo que también incrementa la tasa de amputaciones y muertes en este colectivo ${ }^{4}$. Muchas unidades de úlceras y unidades de pie diabético se han visto cerradas en este tiempo, ya que todos los recursos del hospital (incluido su espacio físico) han sido ocupados para tratar pacientes con COVID-19, y estos pacientes han podido ser seguidos o monitorizados en nuestras clínicas. Estas acciones evitan visitas al hospital relacionadas con el síndrome de pie diabético, reduciendo el riesgo de exposición al COVID-19 en el hospital. En este sentido, debemos entender que todos los profesionales sanitarios trabajamos conjuntamente para combatir un enemigo común, el COVID-19, y no debemos minimizar la importancia del papel de los podólogos y de la podología en descargar el sistema 
sanitario y evitar visitas y hospitalizaciones derivadas de complicaciones del pie diabético o de pacientes en grados avanzados de isquemia con gran fragilidad ${ }^{5}$.

El otro motivo por el que nuestra profesión se ha visto también involucrada en esta pandemia ha sido por la aparición de lesiones con asentamiento en el pie, sospechosas de estar producidas por COVID-19. Observaciones, casos clínicos y series de casos ya han sido publicados recientemente ${ }^{6-11}$ sugiriendo que el COVID-19 podría afectar a los pies y miembros inferiores de los pacientes infectados con diversos tipos de lesiones dérmicas que van desde lesiones similares a perniosis hasta lesiones liveloides o necróticas en los miembros inferiores. Es importante señalar que todas estas informaciones y las series de casos presentadas son valoraciones puramente descriptivas y no analíticas hasta la fecha, por lo que, a pesar de que estos hallazgos podrían suponer un importante avance en el diagnóstico de la infección por COVID-19 en casos de pacientes dudosos o asintomáticos, es importante analizar estos hallazgos con especial cautela e investigarlos cuidadosamente. El Consejo General ha optado por el camino más apropiado en este sentido creando un registro de lesiones de colaboración abierta para recabar toda la información posible por parte de la comunidad podológica. Todos los podólogos que encuentren casos de lesiones susceptibles de ser provocadas por COVID-19 podrán reportar estos casos en el registro abierto creado por el Consejo a tal efecto, lo que sin duda ayudará a la mejor filiación y estudio de esta enfermedad. Iniciativas similares ya se han realizado por parte de la Sociedad Española de Dermatología y Venereología, que ya ha publicado sus resultados sobre 375 casos de lesiones dérmicas con posible relación con la infección por COVID-1912. Desde Revista Española de Podología entendemos que este registro creado por el Consejo General es una fenomenal herramienta que ayuda a la comunidad podológica a aportar "su grano de arena" en la lucha contra la pandemia.

Un saludo,

Javier Pascual Huerta Redactor Jefe. Revista Española de Podología

\section{BIBLIOGRAFÍA}

1. Coronavirus Resource Center of Johns Hopkins University \& Medicine. Disponible en: https://coronavirus.jhu.edu/map.html. Acceso: mayo de 2020.

2. Blanchette V, Brousseau-Foley M, Cloutier L. Effect of contact with podiatry in a team approach context on diabetic foot ulcer and lower extremity amputation: systematic review and meta-analysis. J Foot Ankle Res. 2020;13(1). DOI: 10.1186/s13047-020-0380-8.

3. Armstrong DG, Boulton AJM, Bus SA. Diabetic Foot Ulcers and Their Recurrence. N Engl J Med. 2017;376(24):2367-75. DOI: 10.1056/ NEJMra1615439.

4. Rogers LC, Andros G, Caporusso J, Harkless LB, Mills JL Sr, Armstrong DG. Toe and flow: essential components and structure of the amputation prevention team. J Am Podiatr Med Assoc. 2010;100(5):342-8. DOI: $10.7547 / 1000342$.

5. Rogers LC, Lavery LA, Joseph WS, Armstrong DG. All Feet On Deck-The Role of Podiatry During the COVID-19 Pandemic: Preventing hospitalizations in an overburdened healthcare system, reducing amputation and death in people with diabetes. J Am Podiatr Med Assoc. 2020. DOI: 10.7547/20-051.

6. Mazzotta F, Troccoli T. Acute acro-ischemia in the child at the time of COVID-19. Europ J Pediatr Dermatol. 2020;30:1-4.

7. Fernandez-Nieto D, Jimenez-Cauhe J, Suarex-Valle A, Moreno-Arrones OM, Saceda-Corralo D, Arana-Raja A, et al. Characterization of acute acro-ischemic lesions in non-hospitalized patients: a case series of 132 patients during the COVID-19 outbreak. J Am Acad Dermatol. 2020. pii: S0190-9622(20)30709-X. DOI: 10.1016/j.jaad.2020.04.093.

8. Landa N, Mendieta-Eckert M, Fonda-Pascual P, Aguirre T. Chilblain-like lesions on feet and hands during the COVID-19 Pandemic. Int J Dermatol. 2020. DOI: 10.1111/ijd.14937.

9. Kolivras A, Dehavay F, Delplace D, Feoli F, Meiers I, Milone L, et al. Coronavirus (COVID-19) infection-induced chilblains: a case report with histopathological findings. JAAD Case Reports. 2020. DOI: 10.1016/j. jdcr.2020.04.011.

10. Romaní J, Baselga E, Mitjà O, Riera-Martí N, Garbayo P, Vicente A, et al. Lesiones pernióticas y acrales en España durante el confinamiento por COVID: análisis retrospectivo de 12 casos. Act Dermo-Sifiliogr. 2020. DOI: 10.1016/j.ad.2020.04.002.

11. Piccolo V, Neri I, Filippeschi C, Oranges T, Argenziano G, Battarra VC, et al. Chilblain-like lesions during COVID-19 epidemic: a preliminary study on 63 patients. J Eur Acad Dermatol Venereol. 2020. DOI: 10.1111/ jdv. 16526

12. Galván Casas $C$, Català C, Carretero Hernández G, Rodríguez-Jiménez P, Fernández Nieto D, Rodríguez-Villa Lario A, et al. Classification of the Cutaneous Manifestations of COVID-19: A Rapid Prospective Nationwide Consensus Study in Spain With 375 Cases. Br J Dermatol. 2020. DOI: $10.1111 /$ bjd. 19163 . 